\title{
Superconductivity and antiferromagnetism in $\mathrm{Ba}_{0.75} \mathrm{~K}_{0.25} \mathrm{Fe}_{2} \mathrm{As}_{2}$ single crystals as seen by ${ }^{57} \mathrm{Fe}$ Mössbauer spectroscopy
}

\author{
J. Munevar, ${ }^{1}$ H. Micklitz, ${ }^{1}$ J. Agüero, ${ }^{1}$ Guotai Tan, ${ }^{2}$ Chenglin Zhang,,${ }^{2,3}$ Pengcheng Dai, ${ }^{3}$ and E. Baggio-Saitovitch ${ }^{1, *}$ \\ ${ }^{1}$ Centro Brasileiro de Pesquisas Fisicas, Rua Xavier Sigaud 150, Rio de Janeiro, Brazil \\ ${ }^{2}$ Department of Physics and Astronomy, University of Tennessee, Knoxville, Tennessee 37996-1200, USA \\ ${ }^{3}$ Department of Physics and Astronomy, Rice University, Houston, Texas 77005, USA
}

(Received 11 October 2013; revised manuscript received 4 November 2013; published 25 November 2013)

\begin{abstract}
We have performed detailed ${ }^{57} \mathrm{Fe}$ Mössbauer spectroscopy measurements on $\mathrm{Ba}_{0.75} \mathrm{~K}_{0.25} \mathrm{Fe}_{2} \mathrm{As}_{2}$ single-crystal mosaics showing antiferromagnetic ordering below $T_{N}=95 \mathrm{~K}$ with superconductivity below $T_{C}$ around $30 \mathrm{~K}$. Analysis of the Mössbauer spectra shows a decrease in the magnetic hyperfine field but no change in the magnetic volume fraction below $T_{C}$. This indicates coexistence of magnetism and superconductivity in these compounds.

DOI: 10.1103/PhysRevB.88.184514

PACS number(s): 74.70.Xa, 76.80.+y, 74.20.Mn
\end{abstract}

The coexistence of superconductivity (SC) and antiferromagnetism (AF) in the recently discovered iron-based pnictides is a heavily discussed subject. ${ }^{1}$ Since it is the general opinion that both phenomena originate from $\mathrm{Fe}-3 d$ electrons, a competition between these two phenomena may be expected. A decrease in the local magnetic field in muon spin rotation $(\mu \mathrm{SR})$ recently has been observed in polycrystalline samples of $\mathrm{Ba}_{1-x} \mathrm{~K}_{x} \mathrm{Fe}_{2} \mathrm{As}_{2}{ }^{2}$ It has been proposed that this result implies $s^{+-}$pairing of the Cooper pairs, meaning unconventional superconductivity and coexistence of SDW antiferromagnetism and superconductivity. ${ }^{3}$ However, such a decrease has not been observed for the muon precession frequency in Ni-doped $\mathrm{BaFe}_{2} \mathrm{As}_{2} .{ }^{4}$ On the other hand, a decrease in the Bragg intensities for Ni- and Co-doped $\mathrm{BaFe}_{2} \mathrm{As}_{2}$ by neutron scattering measurements has been seen..$^{5-10}$

The Bragg intensities reflect the product of magnetic volume fraction and magnitude of magnetic moments, and the $\mu \mathrm{SR}$ and other local probe techniques measure the magnitude of local magnetic moments. These both results can be understood if there is a decrease in the magnetic volume fraction below $T_{C}$, but not a change in the magnitude of magnetic moments below $T_{C}$. The length scale of coexistence of the two phenomena, SC and AF order, appears to be another decisive parameter: phase separation on a mesoscopic length scale larger than the SC coherence length(about $2 \mathrm{~nm}^{11}$ ), for example, has been proposed for $\mathrm{Ba}_{1-x} \mathrm{~K}_{x} \mathrm{Fe}_{2} \mathrm{As}_{2} .{ }^{12}$ In a recent paper, on the other hand, the coexistence of the two phenomena, $\mathrm{SC}$ and $\mathrm{AF}$ order, in $\mathrm{Ba}_{1-x} \mathrm{~K}_{x} \mathrm{Fe}_{2} \mathrm{As}_{2}$ on a lattice parameter length scale has been claimed. ${ }^{13}$ The length scale of coexistence of the two phenomena, SC and AF order, in the above given iron-pnictides, therefore, is still an unsolved problem. In order to contribute to a better understanding of the above described open questions, we have performed detailed ${ }^{57} \mathrm{Fe}$ Mössbauer studies above and below $T_{C}$ on single-crystal mosaics of $\mathrm{Ba}_{0.75} \mathrm{~K}_{0.25} \mathrm{Fe}_{2} \mathrm{As}_{2}$ to see whether a change in the static Fe moment at $T_{C}$ can be reflected in the local magnetic hyperfine field at the ${ }^{57} \mathrm{Fe}$ nucleus in this compound.

Single-crystal mosaics of $\mathrm{Ba}_{0.75} \mathrm{~K}_{0.25} \mathrm{Fe}_{2} \mathrm{As}_{2}$ were used for Mössbauer studies, formed by thin platelets mounted in circled mosaics with roughly $1 \mathrm{~cm}$ of diameter. The details of crystal growth procedures are published elsewhere. ${ }^{14}$ Mosaics were mounted with $c$ axis perpendicular to the absorber plane and parallel to $14.4 \mathrm{keV} \gamma$ rays from the ${ }^{57} \mathrm{Co}$ source. Mössbauer spectra were taken in a variable-temperature helium cryostat, allowing temperatures between 2 and $300 \mathrm{~K}$. Both Mössbauer source $\left({ }^{57} \mathrm{Co}: \mathrm{Rh}\right)$, moving in a sinusoidal mode, and absorber have been kept at the same temperature. Isomer shifts are given relative to that of $\alpha$-Fe.

The superconducting response of single crystals of $\mathrm{Ba}_{0.75} \mathrm{~K}_{0.25} \mathrm{Fe}_{2} \mathrm{As}_{2}$ was measured in a SQUID magnetometer with an applied field of 30 Oe parallel to the $a b$ plane, taking 2-45 K as temperature range. From this measurement (see Fig. 1) a diamagnetic response related to the onset of superconducting ordering is clearly observed in the zero-field-cooling mode. The superconducting transition temperature is around $33 \mathrm{~K}$, while the superconducting volume fraction estimated from this measurement gives approximately $80 \%$, indicating nearly full superconducting volume.

The ${ }^{57} \mathrm{Fe}$ Mössbauer spectra taken below $T_{N}$, i.e., in the magnetic regime, generally should be fitted within the SDW model with a distribution of magnetic hyperfine fields represented by a Fourier series expansion. ${ }^{15}$ However, as we have shown in another paper on ${ }^{57} \mathrm{Fe}$ Mössbauer spectroscopy in $\mathrm{BaFe}_{2-x} \mathrm{Ni}_{x} \mathrm{As}_{2}$ pnictides, a "two-site model" can satisfactorily fit all spectra below $T_{N}{ }^{4}$ In this "two-site model" a magnetic and a nonmagnetic site are used. Both sites have the same isomer shift and quadrupole splitting, i.e., correspond to the same crystallographic site. The nonmagnetic sites are related to nonmagnetic regions, probably caused by a distortion of the SDW magnetic structure due to doping. However, we should remark that using the simple "two-site model," the nonmagnetic fraction that we obtain from such a fit will give us an upper limit for the nonmagnetic fraction. In the SDW model, part of the magnetic fraction will have very low magnetic field values and, therefore, the nonmagnetic fraction in the SDW model will be somewhat smaller. Despite the simplicity of our model, we will extract information on the interplay of SC and magnetism in this compound, which essentially is the goal of this work.

Mössbauer spectra in the temperature regime from $4.35 \mathrm{~K}$ to higher temperatures are shown in Fig. 2. From the spectra it is clearly observed that the resonance line begins to broaden below $90 \mathrm{~K}$, caused by SDW order of the Fe moments in the $a b$ plane. Above $T_{N},{ }^{57} \mathrm{Fe}$ Mössbauer spectra shown in Fig. 2, exhibit a single line which has been fitted with an unresolved quadrupole doublet. The ${ }^{57} \mathrm{Fe}$ Mössbauer spectra 




FIG. 1. (Color online) Magnetic susceptibility measurements performed on $\mathrm{Ba}_{0.75} \mathrm{~K}_{0.25} \mathrm{Fe}_{2} \mathrm{As}_{2}$ single crystals, with 30 Oe applied field parallel to the $a b$ plane.

taken below $T_{N}$ have been fitted with a nonmagnetic and a magnetic components, having the same isomer shift and quadrupole interaction, and therefore corresponding to the same crystallographic site.

The nonmagnetic component was considered to be an unresolved quadrupole doublet similar to the one of the paramagnetic state, while the magnetic component was fitted

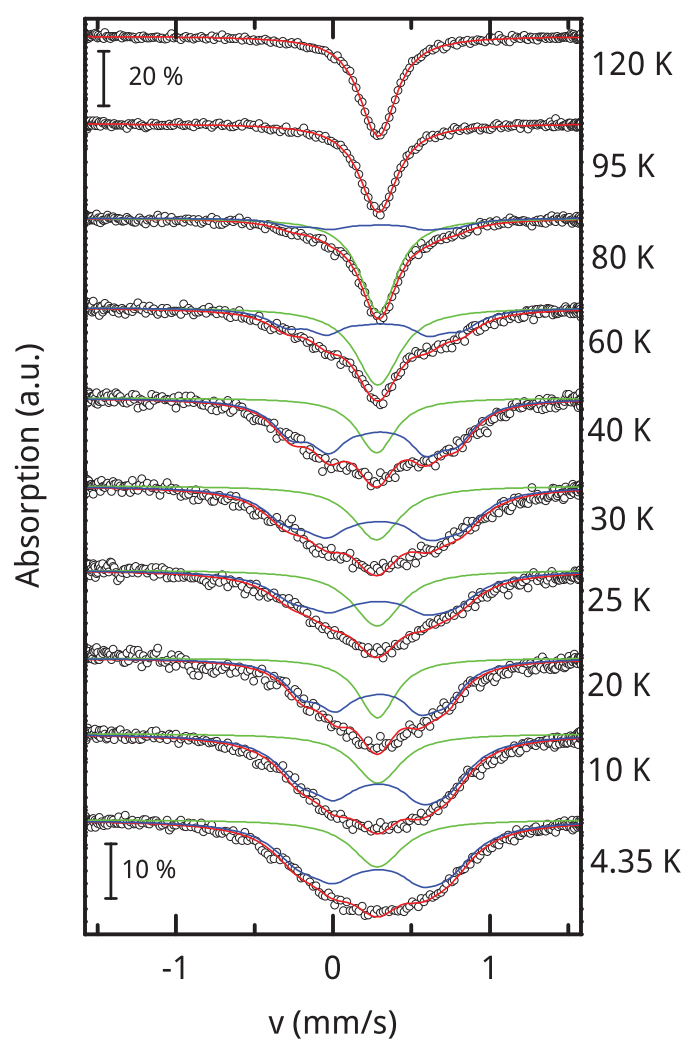

FIG. 2. (Color online) ${ }^{57} \mathrm{Fe}$ Mössbauer spectra for $\mathrm{Ba}_{0.75} \mathrm{~K}_{0.25} \mathrm{Fe}_{2} \mathrm{As}_{2}$ single-crystal mosaics. $\gamma$ ray is parallel to crystal $c$ axis. Fits assuming a two-site model are shown, with a magnetic (blue lines) and a nonmagnetic (green single line) component below $\mathrm{T}_{N}$. within the full Hamiltonian model ${ }^{16}$ essentially having seven fitting parameters such as magnetic hyperfine (hf) field, quadrupole interaction, angle between magnetic hf field and $\gamma$-ray direction (parallel to $c$ axis), as well as that between main component $V_{z z}$ of electric field gradient tensor and $\gamma$-ray direction, isomer shift, linewidth, and line intensity. The direction of $V_{Z Z}$ is parallel to the $c$ axis and $B_{h f}$ is lying in the $a b$ plane; i.e., the angle between $V_{Z Z}$ and $B_{h f}$ is $90^{\circ}$, while that between $V_{Z Z}$ and the $\gamma$-ray direction is zero. We also fixed the linewidth to values obtained in the paramagnetic state.

From the fits described above, we can extract the isomer shift for both sites, the magnetic hyperfine field and the nonmagnetic volume fraction. The isomer shift was found to be $\delta=0.38(1) \mathrm{mm} / \mathrm{s}$, indicating that $\mathrm{Fe}$ is in +2 valence state. Quadrupole splittings are well known for their capability to reflect structural phase transitions affecting the Fe electric interactions. However, we did not find any indication of change in the quadrupole interaction at $T_{N}$, even knowing that a structural transition indeed exists. We have seen a change in the quadrupole splitting at $T_{N}$ for the Ni-doped $\mathrm{BaFe}_{2} \mathrm{As}_{2}$ single crystals. ${ }^{4}$ This may indicate that $\mathrm{K}$ doping induces less disorder in FeAs tetrahedra rather than Ni doping at the $\mathrm{Fe}$ site. Linewidths never exceeded $0.4 \mathrm{~mm} / \mathrm{s}$.

Magnetic hyperfine field $B_{h f}(T)$, magnetic volume fraction, and weighted magnetic hyperfine (hf) field, defined as the product of the magnetic hyperfine field and the magnetic volume fraction, are shown for $\mathrm{Ba}_{0.75} \mathrm{~K}_{0.25} \mathrm{Fe}_{2} \mathrm{As}_{2}$ in Fig. 3 . Magnetic ordering is observed around $95 \mathrm{~K}$ showing a sharp increase of the magnetic moment for Fe. Nevertheless, the magnetic fraction of the sample starts to increase slowly below $T_{N}$, reaching a steady value only below $40 \mathrm{~K}$. This also is observed with $\mu \mathrm{SR}$ for $\mathrm{Ba}_{1-x} \mathrm{~K}_{x} \mathrm{Fe}_{2} \mathrm{As}_{2}$ polycrystalline samples ${ }^{2}$ and in some other iron pnictides for the underdoped regime, ${ }^{17}$ and this may be caused by the doping influence on the local Fe structure. Below $T_{C}(30 \mathrm{~K})$ a decrease in $B_{h f}$ is observed, in agreement with the $\mu \mathrm{SR}$ results on polycrystalline $\mathrm{Ba}_{1-x} \mathrm{~K}_{x} \mathrm{Fe}_{2} \mathrm{As}_{2} .^{2}$ This important finding will be discussed in more detail below. The magnetic moment estimated from $B_{h f}$ using the $6.3 \mathrm{~T} / \mu_{B}$ relation found in Ref. 18 is around $0.48 \mu_{B}$, an indication of itinerant magnetism for Fe due to Fermi surface nesting. ${ }^{3,19,20}$ If we compare this value with that reported for $\mathrm{BaFe}_{2} \mathrm{As}_{2}\left(\mu_{F e}=0.87 \mu_{B}{ }^{18}\right)$ we have to conclude that the decrease in the magnetic moment (or $B_{h f}$ ) is being induced by $\mathrm{K}$ doping on the Ba site, that is, by distortion of the Fermi surface resulting in a reduction of the nesting between hole and electron pockets. The weighted magnetic hf [Fig. 3(b)] field shows a temperature dependence of the $\mathrm{Fe}$ moment looking like an ordinary second-order phase transition. With a microscopic method (Mössbauer) we can distinguish between size of magnetic moment and magnetic volume fraction. With a macroscopic method (magnetization, neutron scattering) one only can measure the product of these 2 quantities. For that reason the magnetic phase transition looks like an ordinary second-order phase transition in neutron scattering experiments, while in our Mössbauer experiments it is more like a local first-order transition, triggered by the structural transition. It should be mentioned that the magnetic volume fraction does not reach $100 \%$ at low temperatures. This in principle is in contradiction with a previous work ${ }^{2}$ where $100 \%$ magnetic volume fraction is reached below some temperature, but we 


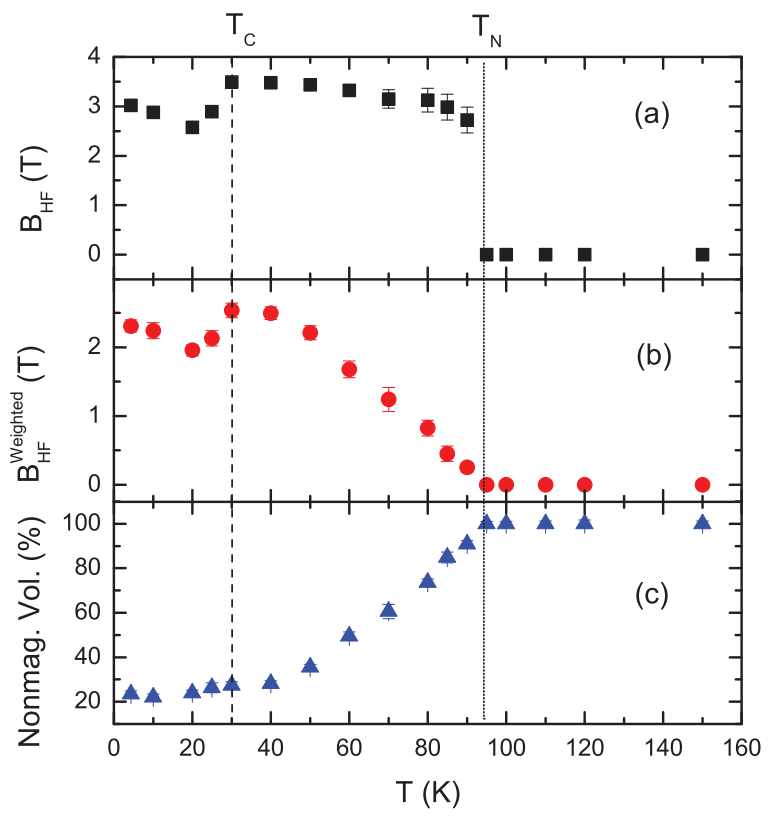

FIG. 3. (Color online) (a) Magnetic hyperfine field $B_{h f}$, (b) weighted $B_{h f}$, and (c) nonmagnetic volume fraction extracted from ${ }^{57} \mathrm{Fe}$ Mössbauer spectra fits for $\mathrm{Ba}_{0.75} \mathrm{~K}_{0.25} \mathrm{Fe}_{2} \mathrm{As}_{2}$ single-crystal mosaics.

notice that this may be an indication of magnetic moments in $\mathrm{Ba}_{0.75} \mathrm{~K}_{0.25} \mathrm{Fe}_{2} \mathrm{As}_{2}$ single crystals that can be sensed by $\mu \mathrm{SR}$ but not by Mössbauer spectroscopy, appearing in the latter as nonmagnetic sites in our simple two-site model (see above).

It is well known that in the iron pnictide systems the Fermi surface for the parent compounds is composed by two concentric electron pockets centered at $(\pi, \pi)$ and two concentric hole pockets centered at $(0,0)$ in the Brillouin zone. Nesting between electron and hole pockets gives rise to SDW order. Superconductivity occurs when nesting is weak, caused by distortion of the Fermi surface through doping or external pressure. ${ }^{19}$ When the system is doped with electrons, the electron pockets expand and the hole pockets contract, and the inverse happens when the system is hole doped. ${ }^{1}$ This Fermi surface distortion reduces nesting and gives possibility to the appearance of Cooper interactions between electrons. ${ }^{20}$ In the light of this information, we can think that for the case in which we have magnetic SDW ordering and superconductivity in the same sample, we can have phase separation with one of the phases still showing Fermi surface nesting and thus magnetic ordering, while for the other phase the Fermi surface nesting can be broken giving rise to superconductivity. On the other hand, we could also expect an overlap between magnetism and superconductivity, which means we would have at the same time conditions to have Fermi surface nesting for weakened magnetism and favorable conditions to have Cooper pairing.

The data presented in Fig. 3 indicate that there is a connection between magnetic ordering and superconductivity: a decrease of the magnetic hyperfine field $B_{h f}$ below $T_{C}$ can be observed. Such a decrease only can be seen if we have either coexistence between magnetism and superconductivity or a phase separation on a length scale smaller than the superconducting coherence length $\zeta_{S C}$. Since $\zeta_{S C} \sim 2 \mathrm{~nm},{ }^{11}$ which is of the order of the unit cell, it obviously does not make sense to talk about a real "phase separation." Coexistence between magnetism and superconductivity, therefore, is indicated from our Mössbauer studies. Neutron scattering studies on the same samples ${ }^{5-8}$ show a decrease in the Bragg peak intensity accompanied by a resonance below $T_{C}$. This means that there is a decrease in the Fe magnetic moment or in the magnetic volume fraction, accompanied by a change in the Fe magnetic moment dynamics. Taking our Mössbauer results which clearly do not see a change in the magnetic volume fraction below $T_{C}$, but a decrease in the magnetic hyperfine field, we can conclude that there is a decrease in the Fe magnetic moment below $T_{C}$. It is argued that such a decrease is caused by a spectral weight transfer when entering the superconducting state. It can be explained by assuming $s^{+-}$ pairing symmetry, where reentrance of the nonmagnetic phase occurs below $T_{C}$ and thus reducing the Fe magnetic moment. ${ }^{3}$

In conclusion we can say that our ${ }^{57} \mathrm{Fe}$ Mössbauer studies indicate the coexistence of magnetism and superconductivity in single crystals of $\mathrm{Ba}_{0.75} \mathrm{~K}_{0.25} \mathrm{Fe}_{2} \mathrm{As}_{2}$ which is seen as a reduction of the $\mathrm{Fe}$ magnetic moment below $T_{C}$. Our data are consistent with NMR measurements, which also show microscopic coexisting AF order and superconductivity. ${ }^{21-23}$ This clearly shows the advantages of using Mössbauer spectroscopy compared to other techniques, e.g., neutron scattering or $\mu \mathrm{SR}$ : (i) ${ }^{57} \mathrm{Fe}$ Mössbauer spectroscopy as a local technique can distinguish between the change of the local magnetic moment and that of the magnetic volume fraction, respectively; this, however, is not possible by neutron scattering; (ii) ${ }^{57} \mathrm{Fe}$ Mössbauer spectroscopy measures the magnetic moment directly at the probe $(\mathrm{Fe})$ atom, while in $\mu \mathrm{SR}$ the site where the muon is coming to rest may be one or several sites away from the Fe position.

This work has been supported by the CNPq (under CIAM collaboration with NSF), CAPES, and the FAPERJ agency in Rio under several projects. The single-crystal growth efforts at UT/Rice were supported by the US DOE, BES, through Contract No. DE-FG02-05ER46202. H.M. acknowledges a visitor fellowship of CAPES and CNPq which supported work at $\mathrm{CBPF}$.

\footnotetext{
*Author to whom correspondences should be addressed: elisa@cbpf.br

${ }^{1}$ P. C. Dai, J. P. Hu, and E. Dagotto, Nat. Phys. 8, 709 (2012).

${ }^{2}$ E. Wiesenmayer, H. Luetkens, G. Pascua, R. Khasanov, A. Amato, H. Potts, B. Banusch, H.-H. Klauss,
}

and D. Johrendt, Phys. Rev. Lett. 107, 237001 (2011)

${ }^{3}$ R. M. Fernandes, D. K. Pratt, W. Tian, J. Zarestky, A. Kreyssig, S. Nandi, M. G. Kim, A. Thaler, N. Ni, P. C. Canfield, R. J. McQueeny, J. Schmalian, and A. I. Goldman, Phys. Rev. B 81, 140501(R) (2010). 
${ }^{4}$ C. Argüello et al. (unpublished).

${ }^{5}$ D. K. Pratt, W. Tian, A. Kreyssig, J. L. Zarestky, S. Nandi, N. Ni, S. L. Bud'ko, P. C. Canfield, A. I. Goodman, and R. J. McQueeney, Phys. Rev. Lett. 103, 087001 (2009).

${ }^{6}$ A. D. Christianson, M. D. Lumsden, S. E. Nagler, G. J. MacDougall, M. A. McGuire, A. S. Sefat, R. Jin, B. C. Sales, and D. Mandrus, Phys. Rev. Lett. 103, 087002 (2009).

${ }^{7}$ M. Wang, H. Luo, J. Zhao, C. Zhang, M. Wang, K. Marty, S. Chi, J. W. Lynn, A. Schneidewind, S. Li, and P. Dai, Phys. Rev. B 81, 174524 (2010).

${ }^{8}$ M. Wang, H. Luo, M. Wang, S. Chi, J. A. Rodriguez-Rivera, D. Singh, S. Chang, J. W. Lynn, and P. Dai, Phys. Rev. B 83, 094516 (2011).

${ }^{9}$ Huiqian Luo, Rui Zhang, Mark Laver, Zahra Yamani, Meng Wang, Xingye Lu, Miaoyin Wang, Yanchao Chen, Shiliang Li, Sung Chang, Jeffrey W. Lynn, and Pengcheng Dai, Phys. Rev. Lett. 108, 247002 (2012).

${ }^{10}$ X. Lu, H. Gretarsson, R. Zhang, X. Liu, H. Luo, W. Tian, M. Laver, Z. Yamani, Y.-J. Kim, A. H. Nevidomskyy, Q. Si, and P. Dai, Phys. Rev. Lett. 110, 257001 (2013).

${ }^{11}$ M. Putti, I. Pallecchi, E. Bellingeri, M. R. Cimberle, M. Tropeano, C. Ferdeghini, A. Palenzona, C. Tarantini, A. Yamamoto, J. Jiang, J. Jaroszynski, F. Kametani, D. Abraimov, A. Polyanskii, J. D. Weiss, E. E. Hellstrom, A. Gurevich, D. C. Larbalestier, R. Jin, B. C. Sales, A. S. Sefat, M. A. McGuire, D. Mandrus, P. Cheng, Y. Jia, H. H. Wen, S. Lee, and C. B. Eom, Supercond. Sci. Technol. 23, 034003 (2010).

${ }^{12}$ J. T. Park, D. S. Inosov, Ch. Niedermayer, G. L. Sun, D. Haug, N. B. Christensen, R. Dinnebier, A. V. Boris, A. J. Drew, L. Schulz,
T. Shapoval, U. Wolff, V. Neu, X. Yang, C. T. Lin, B. Keimer, and V. Hinkov, Phys. Rev. Lett. 102, 117006 (2009).

${ }^{13}$ W. K. Yeoh, B. Gault, X. Y. Cui, C. Zhu, M. P. Moody, L. Li, R. K. Zheng, W. X. Li, X. L. Wang, S. X. Dou, C. T. Lin, and S. P. Ringer, Phys. Rev. Lett. 106, 247002 (2011).

${ }^{14}$ Y. Chen, X. Lu, M. Wang, H. Luo, and S. Li, Supercond. Sci. Technol. 24, 065004 (2011).

${ }^{15}$ A. Olariu, P. Bonville, F. Rullier-Albenque, D. Colson, and A. Forget, New. J. Phys. 14, 053044 (2012).

${ }^{16} \mathrm{~K}$. Ruebenbauer and T. Birchall, Hyperfine Interact. 7, 125 (1979).

${ }^{17}$ A. J. Drew, Ch. Niedermayer, P. J. Baker, F. L. Pratt, S. J. Blundell, T. Lancaster, R. H. Liu, G. Wu, X. H. Chen, I. Watanabe, V. K. Malik, A. Dubroka, M. Rössle, K. W. Kim, C. Baines, and C. Bernhard, Nat. Mater. 8, 310 (2009).

${ }^{18}$ P. Bonville, F. Rullier-Albenque, D. Colson, and A. Forget, Europhys. Lett. 89, 67008 (2010).

${ }^{19}$ R. Chan, M. Gulacsi, A. Ormeci, and A. R. Bishop, Phys. Rev. B 82, 132503 (2010).

${ }^{20} \mathrm{Fa}$ Wang and D. H. Lee, Science 332, 200 (2011).

${ }^{21}$ G. F. Ji, J. S. Zhang, L. Ma, P. Fan, P. S. Wang, J. Dai, G. T. Tan, Y. Song, C. L. Zhang, P. Dai, B. Normand, and W. Yu, Phys. Rev. Lett. 111, 107004 (2013).

${ }^{22}$ S. Avci, O. Chmaissem, D. Y. Chung, S. Rosenkranz, E. A. Goremychkin, J. P. Castellan, I. S. Todorov, J. A. Schlueter, H. Claus, A. Daoud-Aladine, D. D. Khalyavin, M. G. Kanatzidis, and R. Osborn, Phys. Rev. B 85, 184507 (2012).

${ }^{23}$ Z. Li, R. Zhou, Y. Liu, D. L. Sun, J. Yang, C. T. Lin, and Guo-qing Zheng, Phys. Rev. B 86, 180501 (2012). 